\title{
Doubling of the algebra and neutrino mixing within noncommutative spectral geometry
}

\author{
Doubling of the algebra and neutrino mixing
}

\author{
Maria Vittoria Gargiulo ${ }^{1, a}$, Mairi Sakellariadou ${ }^{2, b}$ Giuseppe Vitiello $^{1, \mathrm{c}}$ \\ ${ }^{1}$ Dipartimento di Fisica, I.N.F.N. Università di Salerno, 84100 Salerno, Italy \\ 2 Department of Physics, King's College London, University of London, Strand WC2R 2LS, London, UK
}

Received: 6 September 2013 / Accepted: 9 December 2013 / Published online: 23 January 2014

(C) The Author(s) 2014. This article is published with open access at Springerlink.com

\begin{abstract}
We study the physical implications of the doubling of the algebra, an essential element in the construction of the noncommutative spectral geometry model, proposed by Connes and his collaborators as offering a geometric explanation for the standard model of the strong and electroweak interactions. Linking the algebra doubling to the deformed Hopf algebra, we build Bogoliubov transformations and show the emergence of neutrino mixing.
\end{abstract}

\section{Introduction}

Approaching the Planck energy scale one expects that the notion of a continuous geometrical space ceases to be valid. At such high energy scales the simple hypothesis that physics can be described by the sum of the Einstein-Hilbert action and the standard model (SM) action can no longer be valid. The noncommutative spectral geometry (NCSG) model [1,2] treats the SM as a low-energy phenomenological model which, however, dictates the geometry of spacetime at high energy scales. Hence, the aim of NCSG is to reveal the smallscale structure of spacetime from our knowledge of experimental particle physics at the electroweak scale. Following this approach, to construct a quantum theory of gravity coupled to matter we will consider the gravity-matter interaction to incorporate the most crucial aspect of the dynamics.

At very high energy scales quantum gravity could imply that spacetime is a strongly noncommutative manifold. For energies a few orders of magnitude below the Planck scale, however, it is conceivable that one consider that the algebra of the coordinates can be given by a slightly noncommutative algebra [1-3] which, if appropriately chosen, can lead

\footnotetext{
a e-mail: mariavittoria.gargiulo@gmail.com

b e-mail: mairi.sakellariadou@kcl.ac.uk

c e-mail: vitiello@sa.infn.it
}

to the SM coupled to gravity [4-6]. This slightly noncommutative manifold has been chosen to be the tensor product of an internal (zero-dimensional) Kaluza-Klein (discrete) space and a continuous (four-dimensional) spacetime. Thus, the geometry close to but below the Planck scale is defined by the product $\mathcal{M} \times \mathcal{F}$ of a continuum compact Riemannian manifold $\mathcal{M}$ (for the spacetime) and a discrete finite noncommutative space $\mathcal{F}$ (for the SM) composed by only two points; such a geometry is called almost commutative.

This choice of the doubling of the algebra, which can be interpreted as considering a geometric space formed by two copies (branes) of a four-dimensional manifold, has deep physical implications. As pointed out in Ref. [7], the doubling of the algebra is required to accommodate gauge symmetries, which are necessary to describe the SM, while the doubling of the algebra is also related to dissipation, hence to information loss, thus containing the seeds of quantization.

The purpose of this paper is to show that the doubling of the algebra is also the main element to explain neutrino mixing. Hence, our main motivation is to provide a physical meaning to the mathematical construction of NCSG, a model constructed to give a purely geometric explanation of the SM. The issue of neutrino mixing is particularly important, since it opens interesting perspectives on the physics beyond the SM. Many experimental efforts are thus being made (see for example: http://www.hep.anl.gov/ndk/hypertext/), and the quantum field theory for neutrino mixing (and, in general, for particle mixing) has been formulated [8-18], providing the framework for various studies, also in conjunction with dark energy and dark matter scenarios [19-24].

In what follows, we first give in Sect. 2 a brief presentation of the NCSG elements that we will then use. We summarize in Sect. 3 how neutrinos appear within this construction. In Sect. 4 we relate the algebra doubling, which is a crucial element of the NCSG model, to the Hopf noncommutative 
algebra and Bogoliubov transformations. In Sect. 5 we show how the doubling of the algebra implies neutrino mixing. We then close with our conclusions in Sect. 6.

\section{Elements of NCSG}

Noncommutative spectral geometry, as an approach to unification, is based on three ansatzes, which we state now:

- At some energy level, close to but below the Planck scale, geometry is described by the product of a fourdimensional smooth compact Riemannian manifold, $\mathcal{M}$, with a fixed spin structure, by a discrete noncommutative space, $\mathcal{F}$, composed by only two points.

The noncommutativity of $\mathcal{F}$ can be expressed by a real spectral triple $\left(\mathcal{A}_{\mathcal{F}}, \mathcal{H}_{\mathcal{F}}, D_{\mathcal{F}}\right)$, where $\mathcal{A}_{\mathcal{F}}$ is an involution of operators on the finite-dimensional Hilbert space $\mathcal{H}_{\mathcal{F}}$ of Euclidean fermions, and $\mathcal{D}_{\mathcal{F}}$ is a self-adjoint unbounded operator in $\mathcal{H}_{\mathcal{F}}$. The algebra $\mathcal{A}_{\mathcal{F}}$ contains all information usually carried by the metric. The axioms of the spectral triples imply that the Dirac operator of the internal space, $D_{\mathcal{F}}$, is the fermionic mass matrix. The Dirac operator is the inverse of the Euclidean propagator of fermions. The spectral geometry for $\mathcal{M} \times \mathcal{F}$ is thus given by

$$
\begin{aligned}
\mathcal{A} & =C^{\infty}(\mathcal{M}) \otimes \mathcal{A}_{\mathcal{F}}=C^{\infty}\left(\mathcal{M}, \mathcal{A}_{\mathcal{F}}\right), \\
\mathcal{H} & =\mathcal{L}^{2}(\mathcal{M}, S) \otimes \mathcal{H}_{\mathcal{F}}=\mathcal{L}^{2}\left(\mathcal{M}, S \otimes \mathcal{H}_{\mathcal{F}}\right) \\
D & =D_{\mathcal{M}} \otimes 1+\gamma_{5} \otimes D_{\mathcal{F}},
\end{aligned}
$$

where $C^{\infty}(\mathcal{M}, \mathcal{C})$ is the algebra of smooth complexvalued functions on $\mathcal{M} ; \mathcal{L}^{2}(\mathcal{M}, S)$ is the space of square integrable Dirac spinors over $\mathcal{M} ; D_{\mathcal{M}}$ is the Dirac operator $\not_{\mathcal{M}}=\sqrt{-1} \gamma^{\mu} \nabla_{\mu}^{s}$ on $\mathcal{M}$; and $\gamma_{5}$ is the chirality operator in the four-dimensional case.

- The finite-dimensional algebra $\mathcal{A}_{\mathcal{F}}$, which is the main input, is chosen to be [25]

$$
\mathcal{A}_{\mathcal{F}}=M_{a}(\mathbb{H}) \oplus M_{k}(\mathbb{C}),
$$

with $k=2 a$ and $\mathbb{H}$ being the algebra of quaternions. This choice was made due to the three following reasons:

(1) the model should account for massive neutrinos and neutrino oscillations, so it cannot be a left-right symmetric model, like for instance $\mathbb{C} \oplus \mathbb{H}_{\mathrm{L}} \oplus \mathbb{H}_{\mathrm{R}} \oplus M_{3}(\mathbb{H})$; (2) noncommutative geometry imposes constraints on algebras of operators in the Hilbert space; and (3) one should avoid fermion doubling.

The first possible value for the even number $k$ is 2 , corresponding to a Hilbert space of four fermions, but this choice is ruled out by the existence of quarks. The next possible value is $k=4$, leading to the correct number of $k^{2}=16$ fermions in each of the three generations. This is the most economical choice [26] that can account for the SM.

- The action functional is dictated by the spectral action principle, which affirms that the bosonic part of the action functional depends only on the spectrum of the Dirac operator $\mathcal{D}$ and which is of the form

$\operatorname{Tr}\left(f\left(\frac{\mathcal{D}}{\Lambda}\right)\right)$

where $f$ is a positive even function of the real variable; it falls to zero for large values of its argument. The parameter $\Lambda$ fixes the energy scale. Thus, the action functional sums up eigenvalues of the Dirac operator which are smaller than the cutoff scale $\Lambda$. Since the bosonic action only depends on the spectrum of the line element, i.e. the inverse of the Dirac operator, the operator $\mathcal{D}$ contains all information as regards the bosonic part of the action.

The trace, Eq. (2), is then evaluated with heat kernel techniques and is given in terms of geometrical Seeley-deWitt coefficients $a_{n}$. Since $f$ is a cutoff function, its Taylor expansion at zero vanishes. Therefore, its asymptotic expansion depends only on the three momenta $f_{0}, f_{2}$, and $f_{4}$, which are related to the coupling constant at unification, the gravitational constant, and the cosmological constant, respectively. In this sense, the choice of the test function $f$ plays only a limited rôle. Hence,

$\operatorname{Tr}\left(f\left(\frac{\mathcal{D}}{\Lambda}\right)\right) \sim 2 \Lambda^{4} f_{4} a_{0}+2 \Lambda^{2} f_{2} a_{2}+f_{0} a_{4}$,

where

$f_{k}=\int_{0}^{\infty} f(u) u^{k-1} \mathrm{~d} u$.

The gravitational Einstein action is thus obtained by the expansion of the action functional.

The coupling with fermions is obtained by adding to the trace, Eq. (2), the term

$\operatorname{Tr} \frac{1}{2}\langle J \psi, \mathcal{D} \psi\rangle$,

where $J$ is the real structure on the spectral triple and $\psi$ is an element in the space $\mathcal{H}_{\mathcal{F}}$.

In the presence of gauge fields $A$, there is a modification in the metric (within noncommutative geometry, one does not focus on $g_{\mu \nu}$ but on the Dirac operator instead), leading to inner fluctuations of the metric,

$\mathcal{D} \rightarrow \mathcal{D}_{A}=\mathcal{D}+A+\epsilon^{\prime} J A J^{-1}$,

where $A$ is a self-adjoint operator of the form

$A=\sum_{j} a_{j}\left[D, b_{j}\right], \quad a_{j}, b_{j} \in \mathcal{A}$, 
$J$ is an antilinear isometry, and $\epsilon^{\prime} \in\{-1,1\}$. Applying the action principle to $\mathcal{D}_{A}$ one obtains the combined EinsteinYang-Mills action. Thus, the fermions of the SM provide the Hilbert space of a spectral triple for a suitable algebra, while the bosons arise as inner fluctuations of the corresponding Dirac operator.

In conclusion, the full Lagrangian of the SM, minimally coupled to gravity, is obtained as the asymptotic expansion (in inverse powers of $\Lambda$ ) of the spectral action for the product geometry $\mathcal{M} \times \mathcal{F}$. This geometric model can explain the SM phenomenology [4,6,26,27]. Moreover, since this model lives by construction at very high energies, it can provide a natural framework to address early-universe cosmological issues [28-36].

\section{Neutrinos within the NCSG model}

In the context on NCSG, neutrinos appear naturally as Majorana spinors (so that neutrinos are their own antiparticles), for which the mass terms in the Lagrangian can be written as

$\frac{1}{2} \sum_{\lambda \kappa} \bar{\psi}_{\lambda \mathrm{L}} \mathcal{S}_{\lambda \kappa} \hat{\psi}_{\kappa \mathrm{R}}+\frac{1}{2} \sum_{\lambda \kappa} \overline{\bar{\psi}_{\lambda \mathrm{L}} \mathcal{S}_{\lambda \kappa} \hat{\psi}_{\kappa \mathrm{R}}}$

where the subscripts $L, R$ stand for left-handed and righthanded states, respectively. The off-diagonal parts of the symmetric matrix $\mathcal{S}_{\lambda \kappa}$ are the Dirac mass terms, while the diagonal ones are the Majorana mass terms.

Within NCSG, one can show [2] the existence of a Dirac operator $\mathcal{D}_{\mathcal{F}}$ for the algebra

$$
\begin{aligned}
\mathcal{A}_{\mathcal{F}} & =\left\{\left(\lambda, q_{\mathrm{L}}, \lambda, m\right) \mid \lambda \in \mathbb{C}, q_{\mathrm{L}} \in \mathbb{H}, m \in M_{3}(\mathbb{C})\right\} \\
& \sim \mathbb{C} \oplus \mathbb{H} \oplus M_{3}(\mathbb{C}),
\end{aligned}
$$

with off-diagonal terms. In particular, one can show [2] that there exist $3 \times 3$ matrices ( 3 for the number of generations) $\Upsilon_{e}, \Upsilon_{\nu}, \Upsilon_{d}, \Upsilon_{u}$ and a symmetric $3 \times 3$ matrix (3 for the number of generations) $\Upsilon_{\mathrm{R}}$, such that $\mathcal{D}_{\mathcal{F}}$ is of the form

$\mathcal{D}_{\mathcal{F}}(\Upsilon)=\left(\begin{array}{ll}S & T^{\star} \\ T & \bar{S}\end{array}\right)$.

$S$ is a linear map

$S=S_{1} \oplus\left(S_{\mathrm{q}} \otimes 1_{3}\right)$,

with $1_{3}$ the identity $3 \times 3$ matrix and

$S_{1}=\left(\begin{array}{llll}0 & 0 & \Upsilon_{v}^{\star} & 0 \\ 0 & 0 & 0 & \Upsilon_{e}^{\star} \\ \Upsilon_{\nu} & 0 & 0 & 0 \\ 0 & \Upsilon_{e} & 0 & 0\end{array}\right), S_{\mathrm{q}}=\left(\begin{array}{llll}0 & 0 & \Upsilon_{u}^{\star} & 0 \\ 0 & 0 & 0 & \Upsilon_{d}^{\star} \\ \Upsilon_{u} & 0 & 0 & 0 \\ 0 & \Upsilon_{d} & 0 & 0\end{array}\right) ;$

the subscripts ${ }_{\mathrm{q}}$ and ${ }_{1}$ stand for quarks and leptons, respectively. The ${ }^{\star}$ denotes adjoints and $\bar{S}=\bar{S}_{1} \oplus\left(1_{3} \otimes \bar{S}_{\mathrm{q}}\right)$ act on $\mathcal{H}_{\bar{f}}$ by the complex conjugate matrices; we have splitted $\mathcal{H}_{\mathcal{F}}$ according to $\mathcal{H}_{\mathcal{F}}=\mathcal{H}_{f} \oplus \mathcal{H}_{\bar{f}}$. Finally, $T$ a is linear map, so that $T\left(v_{\mathrm{R}}\right)=\Upsilon_{\mathrm{R}} \bar{\nu}_{\mathrm{R}}$.

The presence of the symmetric matrix $\Upsilon_{R}$ in the Dirac operator of the finite geometry $\mathcal{F}$ accounts for the Majorana mass terms, while $\Upsilon_{\nu}$ is the neutrino Dirac mass matrix. Hence, the restriction of $\mathcal{D}_{\mathcal{F}}(\Upsilon)$ to the subspace of $\mathcal{H}_{\mathcal{F}}$ with the $\left(v_{\mathrm{R}}, v_{\mathrm{L}}, \bar{\nu}_{\mathrm{R}}, \bar{\nu}_{\mathrm{L}}\right)$ basis can be written as a matrix [2]:

$$
\left(\begin{array}{cccc}
0 & M_{v}^{\star} & M_{\mathrm{R}}^{\star} & 0 \\
M_{\nu} & 0 & 0 & 0 \\
M_{\mathrm{R}} & 0 & 0 & \bar{M}_{v}^{\star} \\
0 & 0 & \bar{M}_{\nu} & 0
\end{array}\right)
$$

where $M_{v}=(2 M / g) K_{v}$ with

$2 M=\left[\frac{\operatorname{Tr}\left(\Upsilon_{v}^{\star} \Upsilon_{v}+\Upsilon_{e}^{\star} \Upsilon_{e}+3\left(\Upsilon_{u}^{\star} \Upsilon_{u}+\Upsilon_{d}^{\star} \Upsilon_{d}\right)\right.}{2}\right]^{1 / 2}$,

$K_{v}$ is the neutrino Dirac mass matrix and $M_{\mathrm{R}}$ the Majorana mass matrix.

The equations of motion of the spectral action imply that the largest eigenvalue of $M_{\mathrm{R}}$ is of the order of the unification scale. The Dirac mass $M_{v}$ turns out to be of the order of the Fermi energy, and thus it is much smaller. In conclusion, by the way the NCSG model has been built, it can account for neutrino mixing and the seesaw mechanism.

In the next section we will discuss the links between the NCSG doubling of the algebra and the deformed Hopf algebra and we will show how to obtain the Bogoliubov transformations from linear combinations of deformed coproducts in the Hopf algebra. The neutrino mixing in the context of NCSG will then be discussed in Sect. 5. Mixing will appear to be implied by the doubling of the algebra which is the core of the Connes construction. The neutrino mixing thus appears to be a manifestation of the spectral geometry nature of the construction.

\section{Algebra doubling, Hopf noncommutative algebra, and Bogoliubov transformations}

Let us consider [2] the finite geometry $\mathcal{F}$ described by

$\mathcal{F}=\left(\mathcal{A}_{\mathcal{F}}, \mathcal{H}_{\mathcal{F}}, D_{\mathcal{F}}, J_{\mathcal{F}}, \gamma_{\mathcal{F}}\right)$,

where $J_{\mathcal{F}}$ is an antilinear isometry and $\gamma_{\mathcal{F}}$ is the $\mathbb{Z} / 2$-grading on $\mathcal{M}_{\mathcal{F}}$. The pair $\left(J_{\mathcal{F}}, \gamma_{\mathcal{F}}\right)$ satisfies

$J_{\mathcal{F}}^{2}=1, \quad J_{\mathcal{F}} \gamma_{\mathcal{F}}=-\gamma_{\mathcal{F}} J_{\mathcal{F}}$.

Then consider the product of the finite noncommutative geometry $\mathcal{F}$, with the spectral triple associated with the commutative geometry of a compact four-dimensional Riemannian spin manifold of spacetime $\mathcal{M}$. Note that for a compact spin four-manifold $\mathcal{M}$, the associated spectral triple is 
$\left(C^{\infty}(\mathcal{M}), L^{2}(\mathcal{M}, S), \partial_{\mathcal{M}}\right)$. The product geometry $\mathcal{M} \times \mathcal{F}$ is the real spectral triple [2] (see also Sect. 2)

$$
\begin{aligned}
& (\mathcal{A}, \mathcal{H}, \mathcal{D}, J, \gamma)=\left(C^{\infty}(\mathcal{M}), L^{2}(\mathcal{M}, S), \nexists_{\mathcal{M}}, J_{\mathcal{M}}, \gamma_{5}\right) \\
& \quad \otimes\left(\mathcal{A}_{\mathcal{F}}, \mathcal{H}_{\mathcal{F}}, D_{\mathcal{F}}, J_{\mathcal{F}}, \gamma_{\mathcal{F}}\right)
\end{aligned}
$$

defined as

$$
\begin{aligned}
(\mathcal{A}, \mathcal{H}, \mathcal{D}, J, \gamma)= & \left(\mathcal{A}_{1}, \mathcal{H}_{1}, \mathcal{D}_{1}, J_{1}, \gamma_{1}\right) \\
& \otimes\left(\mathcal{A}_{2}, \mathcal{H}_{2}, \mathcal{D}_{2}, J_{2}, \gamma_{2}\right)
\end{aligned}
$$

with

$$
\begin{aligned}
& \mathcal{A}=\mathcal{A}_{1} \otimes \mathcal{A}_{2}, \mathcal{H}=\mathcal{H}_{1} \otimes \mathcal{H}_{2}, \\
& \mathcal{D}=\mathcal{D}_{1} \otimes 1+\gamma_{1} \otimes \mathcal{D}_{2}, \\
& \gamma=\gamma_{1} \otimes \gamma_{2}, \quad J=J_{1} \otimes J_{2},
\end{aligned}
$$

and

$$
J^{2}=-1, \quad[J, \mathcal{D}]=0, \quad\left[J_{1}, \gamma_{1}\right]=0, \quad\{J, \gamma\}=0,
$$

where square and curl brackets denote commutators and anticommutators, respectively. Note that the resulting geometry $(\mathcal{A}, \mathcal{H}, D, J, \gamma)$ is of KO-dimension $10=2$ modulo 8 . The difference between the two algebras $\mathcal{A}_{1}$ and $\mathcal{A}_{2}$ is that in one the multiplication is made 'row by column', while in the other one multiplication is made 'column by row'. These two algebras are related through the conjugation operator [2].

The doubling map, given in Eq. (9), which is the main element of Connes' NCSG, is intimately related to the noncommutative Hopf algebra characterized by the deformed coproduct map. This can be seen by introducing the (standard) notation

$a \otimes 1 \equiv a_{1}, 1 \otimes a \equiv a_{2}$,

with

$\left\{a_{i}, a_{j}\right\}=0=\left\{a_{i}, a_{j}^{\dagger}\right\}, i \neq j, i, j=1,2$,

and observing that the prescription to work in the NCSG two-mode space $\mathcal{H}=\mathcal{H}_{1} \otimes \mathcal{H}_{2}$ is provided by the Hopf noncommutative coproduct operators given by [37]

$$
\begin{aligned}
& \Delta a_{q}=a_{q} \otimes q^{H}+q^{-H} \otimes a_{q}, \\
& \Delta a_{q}^{\dagger}=a_{q}^{\dagger} \otimes q^{H}+q^{-H} \otimes a_{q}^{\dagger}, \\
& \Delta H=H \otimes \mathbb{1}+\mathbb{1} \otimes H, \\
& \Delta N=N \otimes \mathbb{1}+\mathbb{1} \otimes N .
\end{aligned}
$$

The noncommutative Hopf algebra is thus embedded in Connes' construction. Note that noncommutativity is guaranteed by the so-called 'deformation' parameter $q$. The $H$ and $N$ are operators of the algebra (see below). In Eq. (12), we have also used the notation of the $q$-deformed $h_{q}(1 \mid 1)$ fermionic Hopf algebra for the operators $a_{q}$ and $a_{q}^{\dagger}$. We indeed recall that the algebra $h(1 \mid 1)$ is generated by the set of operators $\left\{a, a^{\dagger}, H, N\right\}$ with $\left\{a, a^{\dagger}\right\}=2 H, \quad[N, a]=-a, \quad\left[N, a^{\dagger}\right]=a^{\dagger}$,

and $[H, \cdot]=0$, with $H$ a central operator, constant in each representation. The deformed algebra Hopf algebra $h_{q}(1 \mid 1)$ embedded in Connes' construction is defined by

$$
\left\{a_{q}, a_{q}^{\dagger}\right\}=[2 H]_{q}, \quad\left[N, a_{q}\right]=-a_{q}, \quad\left[N, a_{q}^{\dagger}\right]=a_{q}^{\dagger},
$$

where $[H, \cdot]=0$, with $N_{q} \equiv N$ and $H_{q} \equiv H$, while $[x]_{q}$ is defined by

$$
[x]_{q}=\frac{q^{x}-q^{-x}}{q-q^{-1}} .
$$

The Casimir operator $\mathcal{C}_{q}$ is given by $\mathcal{C}_{q}=N[2 H]_{q}-a_{q}^{\dagger} a_{q}$. In the fundamental representation we have $H=1 / 2$ and the Casimir operator is thus zero, $\mathcal{C}_{q}=0$. Note that the $q$ deformed coproduct definition is such that $\left[\Delta a_{q}, \Delta a_{q}^{\dagger}\right]=$ $[2 \Delta H]_{q}$, etc., namely the $q$-coproduct algebra is isomorphic with the one defined by Eq. (14). Requiring $a, a^{\dagger}$ and $a_{q}, a_{q}^{\dagger}$ to be adjoint operators implies that $q$ can only be of modulus 1 ; hence $q \sim \mathrm{e}^{i \Theta}$. In the fundamental representation $h(1 \mid 1)$ and $h_{q}(1 \mid 1)$ coincide, as happens in the spin $1 / 2$ representation; the differences appear only at the level of the corresponding coproducts (and in the higher spin representations). Also note that for consistency with the coproduct isomorphism, the Hermitian conjugation of the coproduct must be supplemented by the inversion of the two spaces $\mathcal{H}_{1}$ and $\mathcal{H}_{2}$ in the two-mode space $\mathcal{H}$.

In conclusion, we have seen that the noncommutative ( $q$ deformed) Hopf algebra is embedded in the NCSG construction whose central ingredient is the doubling map $\mathcal{A} \rightarrow$ $\mathcal{A}_{1} \otimes \mathcal{A}_{2}$.

We are now ready to show that the $q$-deformed coproduct turns out to be related to the Bogoliubov transformations, a key ingredient in the neutrino mixing formalism (see Sect. 5). By resorting to the result of Ref. [37], let us define the operators $A_{q}$ and $B_{q}$, as

$$
\begin{aligned}
A_{q} & \equiv \frac{\Delta a_{q}}{\sqrt{[2]_{q}}}=\frac{1}{\sqrt{[2]_{q}}}\left(\mathrm{e}^{i \Theta} a_{1}+\mathrm{e}^{-i \Theta} a_{2}\right), \\
B_{q} & \equiv \frac{1}{i \sqrt{[2]_{q}}} \frac{\delta}{\delta \Theta} \Delta a_{q}=\frac{1}{\sqrt{[2]_{q}}}\left(\mathrm{e}^{i \Theta} a_{1}-\mathrm{e}^{-i \Theta} a_{2}\right),
\end{aligned}
$$

obtained from Eq. (12) with $q=q(\Theta) \equiv \mathrm{e}^{i 2 \Theta}$. The anticommutation relations read

$$
\begin{aligned}
& \left\{A_{q}, A_{q}^{\dagger}\right\}=1, \quad\left\{B_{q}, B_{q}^{\dagger}\right\}=1, \\
& \left\{A_{q}, B_{q}\right\}=0, \quad\left\{A_{q}, B_{q}^{\dagger}\right\}=\tan 2 \Theta .
\end{aligned}
$$

Let us then construct the operators

$$
\begin{aligned}
& a(\Theta)=\frac{1}{\sqrt{2}}(A(\Theta)+B(\Theta)), \\
& \tilde{a}(\Theta)=\frac{1}{\sqrt{2}}(A(\Theta)-B(\Theta)),
\end{aligned}
$$


where

$$
\begin{aligned}
& A(\Theta) \equiv \frac{\sqrt{[2]_{q}}}{2 \sqrt{2}}\left[A_{q(\Theta)}+A_{q(-\Theta)}+A_{q(\Theta)}^{\dagger}-A_{q(-\Theta)}^{\dagger}\right] \\
& B(\Theta) \equiv \frac{\sqrt{[2]_{q}}}{2 \sqrt{2}}\left[B_{q(\Theta)}+B_{q(-\Theta)}-B_{q(\Theta)}^{\dagger}+B_{q(-\Theta)}^{\dagger}\right] .
\end{aligned}
$$

Hence,

$$
\begin{aligned}
& a(\Theta)=U(\Theta) a_{1}-i V(\Theta) a_{2}^{\dagger}, \\
& \tilde{a}(\Theta)=U(\Theta) a_{2}+i V(\Theta) a_{1}^{\dagger},
\end{aligned}
$$

with

$\{a(\Theta), \tilde{a}(\Theta)\}=0$,

and

$$
U^{2}(\Theta)+V^{2}(\Theta)=1, U(\Theta)=\cos \Theta, V(\Theta)=\sin \Theta .
$$

The only nonzero anticommutation relations are

$$
\left\{a(\Theta), a^{\dagger}(\Theta)\right\}=1,\left\{\tilde{a}(\Theta), \tilde{a}^{\dagger}(\Theta)\right\}=1 .
$$

Equation (20) is the Bogoliubov transformation of the pair of creation and annihilation operators $\left(a_{1}, a_{2}\right)$ into $(a(\Theta), \tilde{a}(\Theta))$. Equations (18)-(20) show that the Bogoliubov-transformed operators, $a(\Theta)$ and $\tilde{a}(\Theta)$, are linear combinations of the coproduct operators defined in terms of the deformation parameter $q(\Theta)$ and their $\Theta$-derivatives. Notice in Eq. (20) the antilinearity of the tilde conjugation $c \mathcal{O} \rightarrow c^{*} \tilde{\mathcal{O}}$, which reminds us of the antilinearity of the $J$ isometry introduced in Sect. $2 .^{1}$

It is worth noting that, besides our discussion of neutrino mixing, Bogoliubov transformations are also relevant for quantum aspects of the theory. Indeed, they are known to describe the transition among unitarily inequivalent representations of the canonical (anti)commutation relations in quantum field theory (QFT) at finite temperature and are, therefore, a key tool in the description of the non-equilibrium dynamics of symmetry-breaking phase transitions [38-41]. Here we have shown that Bogoliubov transformations are encoded in the very same structure of the algebra doubling of the Connes construction. This links the NCSG construction with the non-equilibrium dynamics of the early universe as well as with elementary particle physics.

In the next section we show that the noncommutative Hopf algebra embedded in the NCSG construction rules the neutrino mixing phenomenon which is thus 'implied' by the same construction.

\footnotetext{
${ }^{1}$ For more details on this and other features of the $q$-deformed Hopf algebra and the Bogoliubov transformation, we refer the reader to Refs. [37,38].
}

\section{Neutrino mixing}

Our aim here is to show how Bogoliubov transformations, and thus the noncommutative Hopf algebraic structure which has been shown above to be embedded in the NCSG construction, may explain neutrino mixing. Hence, neutrino mixing can find its natural setting in the NGSG construction. Our discussion is based on the quantum field theory algebraic structure and is, therefore, of general character, regardless of the number of Euclidean dimensions. Thus, our result applies to Dirac neutrinos [10-17] as well as to Majorana neutrinos [18], and in principle to other cases of particle mixing (such as meson mixing and quark mixing), too [42]. For concreteness, we refer below to Majorana neutrinos [18].

In the context of NCSG, neutrinos appear naturally as Majorana spinors (neutrinos are their own antiparticles). Connes and his collaborators have derived their model after a Wick rotation to Euclidean signature [2]. Since, unlike Dirac spinors, Majorana spinors do not have a Euclidean version, one may think that there is a problem in the context of NCSG model for massive neutrinos. However, as discussed in detail in Ref. [2], one can use a formalism based on the Pfaffian and Grassmann variables to obtain a substitute for the formalism of Majorana spinors in the Euclidean setup.

Let us introduce the Lagrangian

$$
\begin{aligned}
L(x) & =\bar{\psi}_{m}(x)\left(i \not \partial-M_{\mathrm{d}}\right) \psi_{m}(x) \\
& =\bar{\psi}_{f}(x)(i \not \partial-M) \psi_{f}(x),
\end{aligned}
$$

where we use the notation $x \equiv(\mathbf{x}, t)$, while $\psi_{m}^{T}=\left(v_{1}, v_{2}\right)$ denotes the neutrino fields with nonvanishing masses $m_{1}$ and $m_{2}$, respectively, and $\psi_{f}^{T}=\left(v_{e}, v_{\mu}\right)$ stands for the flavor neutrino fields. We denote $M_{\mathrm{d}}=\operatorname{diag}\left(m_{1}, m_{2}\right)$ and

$M=\left(\begin{array}{cc}m_{e} & m_{e \mu} \\ m_{e \mu} & m_{\mu}\end{array}\right)$,

the mass matrices. For simplicity, we consider only two neutrinos; extension to three neutrino fields can easily be done $[8,9]$. The mixing transformations connecting the flavor fields $\psi_{f}$ to the fields $\psi_{m}$ are

$$
\begin{aligned}
& v_{e}(x)=v_{1}(x) \cos \theta+v_{2}(x) \sin \theta, \\
& v_{\mu}(x)=-v_{1}(x) \sin \theta+v_{2}(x) \cos \theta .
\end{aligned}
$$

The field quantization setting is the standard one; the $\psi_{m}$ fields are free fields in the Lehmann-SymanzikZimmermann (LSZ) formalism of QFT and their explicit expressions in terms of creation and annihilation operators, $\alpha$ and $\alpha^{\dagger}$, are

$$
v_{i}(x)=\sum_{r=1,2} \int \frac{d^{3} \mathbf{k}}{(2 \pi)^{\frac{3}{2}}} \mathrm{e}^{i \mathbf{k} \cdot \mathbf{x}}\left[u_{\mathbf{k}, i}^{r}(t) \alpha_{\mathbf{k}, i}^{r}+v_{-\mathbf{k}, i}^{r}(t) \alpha_{-\mathbf{k}, i}^{r \dagger}\right],
$$


where $u_{\mathbf{k}, i}^{r}(t)=\mathrm{e}^{-i \omega_{\mathbf{k}, i} t} u_{\mathbf{k}, i}^{r}, v_{\mathbf{k}, i}^{r}(t)=\mathrm{e}^{i \omega_{\mathbf{k}, i} t} v_{\mathbf{k}, i}^{r}$, while $r$ is the helicity index and $\omega_{\mathbf{k}, i}=\sqrt{\mathbf{k}^{2}+m_{i}^{2}}$ with $i=1,2$. Note that the operator anticommutation relations and the spinor wave functions' orthogonality and completeness relations are the standard ones and we do not report them here for brevity.

Let $G_{\theta}(t)$ denote the generator of the field mixing transformations Eq. (23):

$$
\begin{aligned}
& v_{e}(x)=G_{\theta}^{-1}(t) v_{1}(x) G_{\theta}(t), \\
& v_{\mu}(x)=G_{\theta}^{-1}(t) v_{2}(x) G_{\theta}(t) .
\end{aligned}
$$

It is given by

$$
G_{\theta}(t)=\exp \left[\frac{\theta}{2} \int d^{3} \mathbf{x}\left(v_{1}^{\dagger}(x) v_{2}(x)-v_{2}^{\dagger}(x) v_{1}(x)\right)\right] .
$$

Due to the canonical anticommutation rules one can write $G_{\theta}(t)=\prod_{\mathbf{k}} G_{\theta}^{\mathbf{k}}(t)$. Moreover, in the reference frame where $\mathbf{k}=(0,0,|\mathbf{k}|)$, we have $G_{\theta}^{\mathbf{k}}(t)=\prod_{r} G_{\theta}^{\mathbf{k}, r}(t)$, with

$$
\begin{aligned}
G_{\theta}^{\mathbf{k}, r}(t)= & \exp \left\{\theta \left[\mathrm{U}_{\mathbf{k}}^{*}(\mathrm{t}) \alpha_{\mathbf{k}, 1}^{\mathrm{r} \dagger} \alpha_{\mathbf{k}, 2}^{\mathrm{r}}-\mathrm{U}_{\mathbf{k}}(\mathrm{t}) \alpha_{-\mathbf{k}, 2}^{\mathrm{r} \dagger} \alpha_{-\mathbf{k}, 1}^{\mathrm{r}}\right.\right. \\
& \left.\left.-\epsilon^{r} V_{\mathbf{k}}^{*}(t) \alpha_{-\mathbf{k}, 1}^{r} \alpha_{\mathbf{k}, 2}^{r}+\epsilon^{r} V_{\mathbf{k}}(t) \alpha_{\mathbf{k}, 1}^{r \dagger} \alpha_{-\mathbf{k}, 2}^{r \dagger}\right]\right\},
\end{aligned}
$$

where $\epsilon^{r}=(-1)^{r}$ and

$$
\begin{aligned}
U_{\mathbf{k}}(t) & \equiv\left|U_{\mathbf{k}}\right| \mathrm{e}^{i\left(\omega_{k, 2}-\omega_{k, 1}\right) t}, \\
V_{\mathbf{k}}(t) & \equiv\left|V_{\mathbf{k}}\right| \mathrm{e}^{i\left(\omega_{k, 2}+\omega_{k, 1}\right) t} .
\end{aligned}
$$

For our purpose it is not essential to give here the explicit expression of $\left|U_{\mathbf{k}}\right|$ and $\left|V_{\mathbf{k}}\right|$; the important point is that

$\left|U_{\mathbf{k}}\right|^{2}+\left|V_{\mathbf{k}}\right|^{2}=1$

which guarantees that the mixing transformations preserve the canonical anticommutation relations, i.e. they are canonical transformations. Equation (29) shows that one can always put $\left|U_{\mathbf{k}}\right|^{2} \equiv \cos ^{2} \Theta_{\mathbf{k}}$ and $\left|V_{\mathbf{k}}\right|^{2} \equiv \sin ^{2} \Theta_{\mathbf{k}}$. Using Eq. (27) we define the flavor annihilation operators:

$$
\begin{aligned}
\alpha_{\mathbf{k}, e}^{r} & \equiv G_{\theta}^{-1} \alpha_{-\mathbf{k}, 1}^{r} G_{\theta}(t) \\
& =\cos \theta \alpha_{\mathbf{k}, 1}^{r}+\sin \theta\left(U_{\mathbf{k}}^{*}(t) \alpha_{\mathbf{k}, 2}^{r}+\epsilon^{r} V_{\mathbf{k}}(t) \alpha_{-\mathbf{k}, 2}^{r \dagger}\right), \\
\alpha_{\mathbf{k}, \mu}^{r} & \equiv G_{\theta}^{-1} \alpha_{-\mathbf{k}, 2}^{r} G_{\theta}(t) \\
& =\cos \theta \alpha_{\mathbf{k}, 2}^{r}-\sin \theta\left(U_{\mathbf{k}}^{*}(t) \alpha_{\mathbf{k}, 1}^{r}+\epsilon^{r} V_{\mathbf{k}}(t) \alpha_{-\mathbf{k}, 1}^{r \dagger}\right)
\end{aligned}
$$

and similar relations for the flavor creation operators.

Note that the Bogoliubov coefficients $U_{\mathbf{k}}$ and $V_{\mathbf{k}}$ are related to the noncommutative coproduct maps discussed in Sect. 4 [cf., e.g., Eq. (20)]. In this connection, we remark that the noncommutative coproduct maps are related, not to the mixing angle $\theta$, but to the Bogoliubov angles $\Theta_{\mathbf{k}}$. Moreover, inspection of Eq. (30) shows that the mixing transformations for the creation and annihilation operators produce 'nested' operator rotation and time-dependent Bogoliubov transformations with coefficients $U_{\mathbf{k}}(t)$ and $V_{\mathbf{k}}(t)$. Since deformed coproducts are a basis of the Bogoliubov transformations, we have thus shown that the field mixing ultimately rests on the algebraic structure of the deformed coproduct in the noncommutative Hopf algebra embedded in the algebra doubling of NCSG. Indeed, for vanishing value of $\left|V_{\mathbf{k}}\right|$, i.e. for vanishing $\Theta_{\mathbf{k}}$ for any $\mathbf{k}$, and thus $\left|U_{\mathbf{k}}\right|^{2}=1$, there is only the field rotation [cf. Eqs. (27) and (30)], not the mixing phenomenon. Of course, the field rotation in the $v_{1}-v_{1}$ plane is a unitary transformation out of which no 'new' quantum number, such as the flavor (lepton) number of $v_{e}$ and $v_{\mu}$, can be generated, as it instead happens in the field mixing case. This result, as already mentioned above, holds for the mixing of any particle considered: Dirac and Majorana neutrinos, quark or meson mixing.

We can finally express the flavor fields in terms of these flavor annihilation and creation operators as [8-17]

$v_{\sigma}(x)=\sum_{r=1,2} \int \frac{d^{3} \mathbf{k}}{(2 \pi)^{\frac{3}{2}}} \mathrm{e}^{i \mathbf{k} \cdot \mathbf{x}}\left[u_{\mathbf{k}, j}^{r}(t) \alpha_{\mathbf{k}, \sigma}^{r}+v_{-\mathbf{k}, j}^{r}(t) \alpha_{-\mathbf{k}, \sigma}^{r \dagger}\right]$,

with $\sigma, j=(e, 1),(\mu, 2)$.

The flavor vacuum annihilated by the operators $\alpha_{\mathbf{k}, \sigma}^{r}, \sigma=$ $e, \mu$, is defined by the action of the mixing generator on the vacuum $|0\rangle_{1,2}$ annihilated by the operators $\alpha_{\mathbf{k}, i}^{r}, i=1,2$, $\left(\alpha_{\mathbf{k}, 1}^{r}|0\rangle_{1,2}=0=\alpha_{\mathbf{k}, 2}^{r}|0\rangle_{1,2}\right)$ as

$|0(\theta, t)\rangle_{e, \mu} \equiv G_{\theta}^{-1}(t)|0\rangle_{1,2}$.

The expectation value of the number operator $\alpha_{\mathbf{k}, i}^{r \dagger} \alpha_{\mathbf{k}, i}^{r}, i=$ 1,2 , in such a vacuum state $|0(\theta, t)\rangle_{e, \mu}$ is nonzero, i.e.,

${ }_{e, \mu}\left\langle 0(t)\left|\alpha_{\mathbf{k}, i}^{r \dagger} \alpha_{\mathbf{k}, i}^{r}\right| 0(t)\right\rangle_{e, \mu}=\left|V_{\mathbf{k}}(t)\right|^{2} \sin ^{2}(\theta), \quad i=1,2$,

which expresses that the flavored vacuum is a condensate (of couples) of $i$-neutrinos, $i=1,2$, hence its nonperturbative nature. We see that the expectation value of the number operator vanishes in the $\left|V_{\mathbf{k}}(t)\right| \rightarrow 0$ limit, i.e. in the commutative limit where the Bogoliubov transformations are eliminated [cf. Eq. (30)]. We remark that the space of the neutrino flavored states is unitarily inequivalent to the space of the mass neutrino eigenstates. Indeed, in the limit of the volume $V$ going to infinity, one obtains

${ }_{1,2}\langle 0 \mid 0(t)\rangle_{e, \mu} \rightarrow 0, \quad$ as $V \rightarrow \infty$ for any $t$,

which shows that $|0(t)\rangle_{e, \mu}$ and $|0(t)\rangle_{1,2}$ are unitarily inequivalent representations of the canonical anticommutator relations. In the absence of mixing $\left(\theta=0\right.$ and/or $\left.m_{1}=m_{2}\right)$ the orthogonality between $|0(t)\rangle_{e, \mu}$ and $|0(t)\rangle_{1,2}$ disappears. Equation (34) can only hold in the QFT framework, since unitarily inequivalent representations exist, contrarily to what 
happens in quantum mechanics (QM), where the von Neumann theorem states the unitary equivalence of the representations of the canonical anticommutation relations. Equation (34) also expresses the nonperturbative nature of the field mixing mechanism.

The single (mixed) particle flavored state is given by

$\left|\alpha_{\mathbf{k}, \sigma}^{r}(t)\right\rangle \equiv \alpha_{\mathbf{k}, \sigma}^{r \dagger}(t)|0(t)\rangle_{e, \mu}=G_{\theta}^{-1}(t) \alpha_{\mathbf{k}, i}^{r \dagger}|0\rangle_{1,2}$,

where $\sigma, i=e, 1$ or $\mu, 2$. States with particle number higher than 1 are obtained similarly by operating repeatedly with the creation operator $\alpha_{\mathbf{k}, \sigma}^{r \dagger}$. The momentum operator for the free fields is

$\mathbf{P}_{i}=\sum_{r=1,2} \int d^{3} \mathbf{k} \mathbf{k}\left(\alpha_{\mathbf{k}, i}^{r \dagger} \alpha_{\mathbf{k}, i}^{r}-\alpha_{-\mathbf{k}, i}^{r \dagger} \alpha_{-\mathbf{k}, i}^{r}\right)$,

with $i=1,2$. For mixed fields, one has $\mathbf{P}_{\sigma}(t)=$ $G_{\theta}^{-1}(t) \mathbf{P}_{i} G_{\theta}(t)$, namely

$$
\begin{aligned}
\mathbf{P}_{\sigma}(t)= & \sum_{r=1,2} \int d^{3} \mathbf{k} \mathbf{k}\left(\alpha_{\mathbf{k}, \sigma}^{r \dagger}(t) \alpha_{\mathbf{k}, \sigma}^{r}(t)\right. \\
& \left.-\alpha_{-\mathbf{k}, \sigma}^{r \dagger}(t) \alpha_{-\mathbf{k}, \sigma}^{r}(t)\right),
\end{aligned}
$$

for $\sigma=e, \mu$ with $\mathbf{P}_{e}(t)+\mathbf{P}_{\mu}(t)=\mathbf{P}_{1}+\mathbf{P}_{2} \equiv \mathbf{P}$ and $\left[\mathbf{P}, G_{\theta}(t)\right]=0$. The total momentum is of course conserved, $[\mathbf{P}, H]=0$, with $H$ denoting the Hamiltonian. The expectation value of the flavor vacuum of the momentum operator $\mathbf{P}_{\sigma}(t)$ vanishes at all times:

${ }_{e, \mu}\left\langle 0(t)\left|\mathbf{P}_{\sigma}(\mathbf{t})\right| 0(t)\right\rangle_{e, \mu}=0, \quad \sigma=e, \mu$.

The state $\left|\alpha_{\mathbf{k}, e}^{r}\right\rangle \equiv\left|\alpha_{\mathbf{k}, e}^{r}(0)\right\rangle$ is an eigenstate of the momentum operator $\mathbf{P}_{e}(0)$ at time $t=0, \mathbf{P}_{e}(0)\left|\alpha_{\mathbf{k}, e}^{r}\right\rangle \equiv \mathbf{k}\left|\alpha_{\mathbf{k}, e}^{r}\right\rangle$. At time $t \neq 0$ the normalized expectation value for the momentum in such a state is

$$
\begin{aligned}
\mathcal{P}_{\mathbf{k}, \sigma}^{e}(t) & \equiv \frac{\left\langle\alpha_{\mathbf{k}, e}^{r}\left|\mathbf{P}_{\sigma}(t)\right| \alpha_{\mathbf{k}, e}^{r}\right\rangle}{\left\langle\alpha_{\mathbf{k}, e}^{r}\left|\mathbf{P}_{\sigma}(0)\right| f \alpha_{\mathbf{k}, e}^{r}\right\rangle} \\
& =\left|\left\{\alpha_{\mathbf{k}, e}^{r}(t), \alpha_{\mathbf{k}, e}^{r \dagger}\left(t^{\prime}\right)\right\}\right|^{2}+\left|\left\{\alpha_{-\mathbf{k}, e}^{r \dagger}(t), \alpha_{\mathbf{k}, e}^{r \dagger}\left(t^{\prime}\right)\right\}\right|^{2},
\end{aligned}
$$

for $\sigma=e, \mu$.

Note that $\mathcal{P}_{\mathbf{k}, \sigma}^{e}(t)$ behaves actually as a 'charge operator'. Indeed, the operator $\alpha_{\mathbf{k}, i}^{r \dagger} \alpha_{\mathbf{k}, i}^{r}-\alpha_{-\mathbf{k}, i}^{r \dagger} \alpha_{-\mathbf{k}, i}^{r}$ is the fermion number operator. Therefore, the explicit calculation of $\mathcal{P}_{\mathbf{k}, \sigma}^{e}(t)$ provides the flavor charge oscillation. We obtain

$$
\begin{aligned}
& \mathcal{P}_{\mathbf{k}, e}^{e}(t)=1-\sin ^{2} 2 \theta \\
& \quad \times\left[\left|U_{\mathbf{k}}\right|^{2} \sin ^{2} \frac{\omega_{k, 2}-\omega_{k, 1}}{2} t+\left|V_{\mathbf{k}}\right|^{2} \sin ^{2} \frac{\omega_{k, 2}+\omega_{k, 1}}{2} t\right],
\end{aligned}
$$

$$
\begin{aligned}
& \mathcal{P}_{\mathbf{k}, \mu}^{e}(t)=\sin ^{2} 2 \theta \\
& \quad \times\left[\left|U_{\mathbf{k}}\right|^{2} \sin ^{2} \frac{\omega_{k, 2}-\omega_{k, 1}}{2} t+\left|V_{\mathbf{k}}\right|^{2} \sin ^{2} \frac{\omega_{k, 2}+\omega_{k, 1}}{2} t\right] .
\end{aligned}
$$

Notice that in the absence of the condensate contribution, i.e. in the $\left|V_{\mathbf{k}}\right| \rightarrow 0$ limit $\left(\left|U_{\mathbf{k}}\right| \rightarrow 1\right)$, the usual QM Pontecorvo approximation of the oscillation formula is obtained. In the same limit, the noncommutative structure of the Hopf coproduct algebra (and the related Bogoliubov transformation) is lost. The nonperturbative structure of the quantum field is thus essential for the NCSG construction.

\section{Conclusions}

We have shown that neutrino mixing is naturally embedded within the NCSG model. This result has been obtained from the doubling of the algebra $\mathcal{A}=\mathcal{A}_{1} \otimes \mathcal{A}_{2}$ acting on the space $\mathcal{H}=\mathcal{H}_{1} \otimes \mathcal{H}_{2}$. In fact, by considering the neutrino mixing, we have seen in Section $\mathrm{V}$ that the transformation linking mass annihilation and creation operators with the flavor ones is a rotation combined ('nested') with Bologiubov transformations [cf. Eq. (30)]. This transformation is the seed of the mixing annihilation and creation operators leading to the unitarily inequivalence between the two vacuum states, i.e. mass vacuum and flavor vacuum. In Section IV we have shown that the Bogoliubov transformed operators, $a(\Theta)$ and $\tilde{a}(\Theta)$, are linear combinations of the coproduct operators defined in terms of the deformation parameter $q(\Theta)$ and its $\Theta$-derivatives, obtained from the doubled algebra $\mathcal{A}=\mathcal{A}_{1} \otimes \mathcal{A}_{2}$. Neutrino mixing is thus intimately related to the algebra doubling and, as such, it is intrinsically present in the NCSG of model.

We stress that Bogoliubov transformations act on operators, so our discussion is framed in the quantum operator formalism. Thus, the doubling of the algebra in Connes' construction appears to be grounded in the QFT Hopf deformed algebra, and in turn this has been shown to involve field mixing. Having to do with fields introduces crucial features in the formalism. On the one side, it means that we have an infinite number of degrees of freedom (therefore, we have to consider the continuum or the infinite volume limit). On the other side, as emerges from the discussion presented above, the algebra doubling, through the Bogoliubov transformations, combines the positive frequency part of the field operator with the negative frequency one, leading to the noncommutative features.

It has been shown in Ref. [7] that the gauge structure of the Standard Model is implicit in the algebra doubling, a key ingredient of the NCSG construction. In the present paper we have established the link between the algebra doubling and the field mixing, concluding that the Standard Model as 
derived from the NCSG model includes neutrino mixing by construction.

Open Access This article is distributed under the terms of the Creative Commons Attribution License which permits any use, distribution, and reproduction in any medium, provided the original author(s) and the source are credited.

Article funded by SCOAP ${ }^{3}$ and licensed under CC BY 4.0

\section{References}

1. A. Connes, Noncommutative Geometry (Academic Press, New York, 1994)

2. A. Connes, M. Marcolli, Noncommutative Geometry, Quantum Fields and Motives (Hindustan Book Agency, India, 2008)

3. K. van den Dungen, W.D. van SuijlekomRev, Rev. Math. Phys. 24, 1230004 (2012)

4. A.H. Chamseddine, A. Connes, M. Marcolli, Adv. Theor. Math. Phys. 11, 991 (2007). [arXiv:hep-th/0610241]

5. A. Connes, JHEP 0611, 081 (2006). [arXiv:hep-th/0608226]

6. A.H. Chamseddine, A. Connes, JHEP 1209, 104 (2012). [arXiv: 1208.1030 [hep-ph]]

7. M. Sakellariadou, A. Stabile, G. Vitiello, Phys. Rev. D 84, 045026 (2011). [arXiv:1106.4164 [math-ph]]

8. M. Blasone, G. Vitiello, Ann. Phys. 244, 283 (1995). [arXiv: hep-ph/9501263]

9. M. Blasone, A. Capolupo, G. Vitiello, Phys. Rev. D 66, 025033 (2002)

10. M. Blasone, P.A. Henning, G. Vitiello, Phys. Lett. B 451, 140 (1999). [arXiv:hep-th/9803157]

11. M. Blasone, A. Capolupo, O. Romei, G. Vitiello, Phys. Rev. D 63, $125015(2001)$

12. C.-R. Ji, Y. Mishchenko, Phys. Rev. D 65, 096015 (2002)

13. A. Capolupo, C.-R. Ji, Y. Mishchenko, G. Vitiello, Phys. Lett. B 594, 135 (2004)

14. N. Mavromatos, S. Sarkar, Phys. Rev. D 72, 065016 (2005)

15. N. Mavromatos, S. Sarkar, New J. Phys. 10, 073009 (2008)

16. N. Mavromatos, S. Sarkar, W. Tarantino, Phys. Rev. D 80, 084046 (2009)

17. M. Blasone, M. Di Mauro, G. Vitiello, Phys. Lett. B 697, 238 (2011)

18. M. Blasone, J. Palmer, Phys. Rev. D 69, 057301 (2004)

19. A. Capolupo, S. Capozziello, G. Vitiello, Phys. Lett. A 363, 53 (2007). [arXiv:astro-ph/0602467]
20. A. Capolupo, S. Capozziello, G. Vitiello, Phys. Lett. A 373, 601 (2009). [arXiv:0809.0085 [hep-th]]

21. A. Capolupo, S. Capozziello, G. Vitiello, Int. J. Mod. Phys. A 23, 4979 (2008). [arXiv:0705.0319 [hep-th]]

22. M. Blasone, A. Capolupo, S. Capozziello, G. Vitiello, Nucl. Instrum. Methods A 588, 272 (2008). [arXiv:0711.0939 [hep-th]]

23. M. Blasone, A. Capolupo, G. Vitiello, Prog. Part. Nucl. Phys. 64, 451 (2010). [arXiv:0912.1489 [hep-th]]

24. M. Blasone, A. Capolupo, S. Capozziello, S. Carloni, G. Vitiello, Phys. Lett. A 323, 182 (2004). [arXiv:gr-qc/0402013]

25. A.H. Chamseddine, A. Connes, Phys. Rev. Lett. 99, 191601 (2007). [arXiv:0706.3690 [hep-th]]

26. A. Devastato, F. Lizzi, P. Martinetti. [arXiv:1304.0415 [hep-th]]

27. A.H. Chamseddine, A. Connes, W.D. van Suijlekom. [arXiv:1304 8050 [hep-th]]

28. W. Nelson, M. Sakellariadou, Phys. Rev. D 81, 085038 (2010). [arXiv:0812.1657 [hep-th]]

29. W. Nelson, M. Sakellariadou, Phys. Lett. B 680, 263 (2009). [arXiv: 0903.1520 [hep-th]]

30. M. Marcolli, E. Pierpaoli. [arXiv:0908.3683 [hep-th]]

31. M. Buck, M. Fairbairn, M. Sakellariadou, Phys. Rev. D 82, 043509 (2010). [arXiv:1005.1188 [hep-th]]

32. W. Nelson, J. Ochoa, M. Sakellariadou, Phys. Rev. Lett. 105, 101602 (2010). [arXiv:1005.4279 [hep-th]]

33. W. Nelson, J. Ochoa, M. Sakellariadou, Phys. Rev. D 82, 085021 (2010). [arXiv:1005.4276 [hep-th]]

34. M. Sakellariadou, Introduction and Phenomenological/ Cosmological Consequences

35. M. Sakellariadou. [arXiv:1101.2174 [hep-th]]

36. G. Lambiase, M. Sakellariadou, A. Stabile. [arXiv:1302.2336 [grqc]]

37. E. Celeghini, S. De Martino, S. De Siena, A. Iorio, M. Rasetti, G. Vitiello, Phys. Lett. A 244, 455 (1998). [arXiv:hep-th/9801031]

38. M. Blasone, P. Jizba, G. Vitiello, Quantum Field Theory and its Macroscopic Manifestations (Imperial College, London, 2011)

39. H. Umezawa, H. Matsumoto, M. Tachiki, Thermo Field Dynamics and Condensed States (North-Holland, Amsterdam, 1982)

40. H. Umezawa, Advanced Field Theory: Micro, Macro, and Thermal Physics (AIP, New York, 1993)

41. G. Vitiello, in Topological defects and the non-equilibrium dynamics of symmetry breaking phase transitions, ed. by Y.M. Bunkov, H.Godfrin, NATO Science Series C 549 (Kluwer Academic Publishers, Dordrecht, 2000), p. 171. [arXiv:hep-th/9902139]

42. A. Connes, Int. J. Geom. Methods Mod. Phys. 5, 1215 (2008). [arXiv:0810.2091 [hep-th]] 